\section{Avaliação da implantação de serviços de saúde reprodutiva no Município de Maringá, Paraná, Brasil}

\author{
Evaluation of the implementation of reproductive \\ health services in Maringá, Paraná State, Brazil
}

Elizabeth Eriko Ishida Nagahama ${ }^{1}$

\section{Introdução}

The aim of this study was to develop a tool to evaluate the implementation of a contraceptive program in health services and apply it to the 23 public health services in Maringá, Paraná State, Brazil. A theoretical-logical model was developed, corresponding to a "target image" for the family planning program. Using the Delphi technique and consensus conference, six experts validated the program's target image, which included three dimensions and 60 evaluation criteria. A data collection instrument was prepared, in addition to a spreadsheet to evaluate the degree of the family planning program's implementation, constituting the Questionnaire for the Evaluation of Reproductive Health Services. The vast majority of the primary health units (91.3\%) received an "intermediate" score on implementation of the family planning program, while $8.7 \%$ were classified as "incipient" and none were scored as "advanced". The "advanced" degree of implementation in the structural dimension contrasted with the organizational and patient care dimensions. The instrument can be useful for evaluating reproductive health programs and is applicable to the health services planning and management processes.

Health Management; Reproductive Health Services; Health Evaluation; Family Planning
Na Conferência Internacional sobre População e Desenvolvimento, realizada no Cairo, Egito, em 1994, um acordo entre 179 países outorgou um papel primordial à saúde e aos direitos sexuais e reprodutivos, sendo uma das suas principais metas o acesso universal à saúde reprodutiva até $2015^{1,2}$

No Brasil, a implantação do Programa de Atenção Integral à Saúde da Mulher (PAISM) em 1984, incorporou o planejamento familiar às ações de atenção integral à saúde da mulher. Em 2001, a Norma Operacional de Assistência à Saúde (NOAS) do Ministério da Saúde estabeleceu, entre outras, a assistência em planejamento familiar no elenco de ações mínimas que devem ser implementadas em todos os municípios 1,3. Atualmente, no contexto dos direitos sexuais e reprodutivos, a assistência em planejamento familiar constitui uma das áreas de atuação prioritária do Ministério da Saúde, enunciada na Política Nacional de Direitos Sexuais e Direitos Reprodutivos 4 .

Apesar da existência de política governamental que estabelece diretrizes e regulamenta a implantação e implementação técnica das ações de planejamento familiar no Brasil, a revisão bibliográfica revela a dificuldade de acesso a serviços que oferecem o programa de planejamento familiar, a má qualidade da atenção, a dificuldade de integrar ações e a oferta limitada de métodos 
contraceptivos. Essa inadequação na assistência pode ser expressa, tirante outros exemplos, no conhecimento limitado das mulheres sobre os métodos contraceptivos, no seu uso inadequado, na falta de continuidade e seguimento no manejo de métodos, na desarticulação entre as atividades educativas e clínicas e na relação interpessoal autoritária entre clientes e profissionais de saúde 5,6,7,8,9,10,11,12.

Vieira 7 ressalta que a medicalização e a privatização caracterizam a situação do planejamento familiar no Brasil, com a concentração no uso de dois métodos contraceptivos: a esterilização cirúrgica e a pílula.

Tal padrão de uso dos métodos anticoncepcionais também foi encontrado em inquérito populacional desenvolvido por Souza 13 no Município de Maringá, Paraná, Brasil. A autora apontou que o anticoncepcional hormonal oral (50,3\%), a laqueadura tubária (32\%) e o preservativo masculino $(28,1 \%)$ foram os métodos contraceptivos mais utilizados pelas usuárias do sistema público de saúde. A pouca expressividade $(1,7 \%)$ de outros métodos anticoncepcionais citados na pesquisa (injetável, preservativo feminino, espumas e geléias) e a ausência no uso de métodos como o diafragma, "norplant" ou endoceptivo indicaram o desconhecimento das mulheres sobre o leque de opções para regular a fecundidade, a limitada oferta de métodos e, portanto, resultaram na escolha concentrada em alguns métodos contraceptivos.

Outro estudo realizado no Hospital Universitário de Maringá, Universidade Estadual de Maringá 14 , identificou que os métodos contraceptivos mais utilizados pelos casais que se candidataram à vasectomia foram o anticoncepcional hormonal oral e o preservativo masculino, provavelmente por constituírem os mais divulgados, de baixo custo e acessíveis no serviço público de saúde.

Osis et al. 15 reforçam estas informações ao inferir que a atenção ao planejamento familiar no Brasil continua a ser marcada pela indisponibilidade de métodos anticoncepcionais nos serviços públicos de saúde, pela capacitação desigual e insuficiente dos profissionais e pelo papel secundário que o planejamento familiar ocupa nas ações básicas de saúde.

Esses dados levantaram questionamentos sobre vários aspectos da atenção em contracepção, relacionados à efetiva implantação da assistência em planejamento familiar nas unidades básicas de saúde, à dificuldade de acesso aos serviços e à qualidade da atenção. Além disso, estudos sobre contracepção na rede de serviços municipais são pouco freqüentes e as informações sobre a qualidade da atenção, escassa. Desta forma, confirma-se a distância entre o discurso oficial - avaliação como componente indispensável do processo de gerenciamento e redirecionamento de atividades e procedimentos programados - e a ausência prática deste instrumental de gestão nos serviços de assistência à saúde.

Se, por um lado, a literatura aponta para a precariedade na atenção em planejamento familiar, de outro, ações programáticas raramente foram avaliadas em sua implantação. Poucos estudos avaliativos enfocam as estratégias de implantação e o grau de implantação dos programas de planejamento familiar nos serviços públicos de saúde.

Perante essas informações, questiona-se se a precariedade da atenção em planejamento familiar é justificada pela dificuldade de acesso e má qualidade da atenção ou pelo fato de o programa de planejamento familiar não ter sido, efetivamente, implantado nos serviços públicos de saúde.

Considerando as palavras de McKinlay 16 (p. 241), de que "não só processos determinam resultados, e é relevante analisar em que medida o programa foi adequadamente implementado nas dimensões propostas", e de Hartz 17 (p. 96) "não se pode assegurar a eficácia das intervenções se não se souber antes quais são suas características de implantação, ou seja, o grau de implementação e os fatores que favorecem sua dinâmica interna"; $\mathrm{e}$ a reconhecida importância da atenção em contracepção na qualidade de vida de homens e mulheres, o presente estudo objetivou desenvolver um instrumento para avaliar a implantação da assistência em contracepção em serviços de saúde, bem como aplicá-lo nas vinte e três unidades básicas de saúde no Município de Maringá.

\section{Material e método}

\section{Desenho e local do estudo}

Trata-se de pesquisa avaliativa de análise de implantação, realizada por intermédio de censo nas 23 unidades básicas de saúde no Município de Maringá, no período de julho a agosto de 2006.

O Município de Maringá, localizado no Noroeste do Estado do Paraná, constitui a terceira maior cidade do estado e possui 324.395 habitantes, sendo cidade pólo da $15^{\text {a }}$ Regional de Saúde, a qual engloba 29 municípios. A primeira equipe do Programa Saúde da Família (PSF) foi implantada em 1999 e, atualmente, compõe-se de 61 equipes, com cobertura de $80 \%$ da população 18 .

No ano de 2004, a coordenação da Saúde da Mulher do município realizou a capacitação das 
equipes do PSF e médicos gineco-obstetras, com o objetivo de revisar o protocolo de planejamento familiar 19. No ano de 2006, o município adotou a estratégia de "Municípios e Comunidades Saudáveis" da Organização Pan-Americana da Saúde, como eixo da política pública de saúde. Inserido nessa proposta de reorientação das ações de prevenção em saúde, o subprograma de promoção da saúde da mulher incorporou as ações de planejamento familiar 18 .

\section{Construindo a imagem-objetivo do \\ planejamento familiar: modelo \\ teórico-lógico, conferência de \\ consenso, dimensões e \\ critérios de avaliação}

Para selecionar as dimensões e critérios para a pesquisa avaliativa, foi elaborado um modelo teórico-lógico 20 , correspondente a uma imagemobjetivo do programa de planejamento familiar. A imagem-objetivo contemplou os atributos estruturais e organizacionais necessários para implantação efetiva da assistência ao planejamento familiar, bem como os resultados e impactos esperados do programa. Foi construída tomando-se por base o manual para o gestor do planejamento familiar do Ministério da Saúde 1 e protocolo de planejamento familiar do município 19 (Figura 1).

A imagem-objetivo constituiu referência para apreciar o grau de implantação dos componentes de estrutura e processo do programa e explicitar quais componentes da intervenção foram avaliados.

Foram selecionadas três dimensões para compor a imagem-objetivo do planejamento familiar, denominadas de dimensão estrutural, organizacional e assistencial, sumarizadas numa matriz contendo a descrição do padrão esperado para cada uma das dimensões, o que totalizou 57 critérios de avaliação.

Por meio da utilização da técnica Delphi 21, seis profissionais da área da saúde sexual e reprodutiva foram convidados a manifestar individualmente a sua concordância ou discordância com a proposta de imagem-objetivo do programa, atribuindo, a cada um dos critérios de avaliação, de zero a dez pontos, segundo a importância relativa de cada um deles. Solicitou-se aos especialistas que sugerissem a exclusão, inclusão ou modificação de dimensões e critérios. A planilha foi encaminhada pela pesquisadora e foi devolvida após 30 dias, por correio eletrônico.

As informações contidas nas planilhas foram consolidadas no programa Excel (Microsoft Corp., Estados Unidos). Calculou-se, para cada critério, a média aritmética - quanto maior a média, maior a importância do critério -, e o desvio-padrão, que estimou o grau ou ausência de consenso. Utilizaram-se os pontos de corte estabelecidos no trabalho de Souza et al. ${ }^{21}$, que foram os seguintes: (1) todo critério com média superior a sete foi considerado como importante. Abaixo disso, seria pouco importante, não devendo compor a imagem-objetivo; (2) todo critério com desvio padrão inferior a três foi considerado consensual; e (3) os critérios com média igual ou superior a sete e desvio padrão igual ou superior a três, apesar de importantes, não compuseram a imagem-objetivo, pois não foram consensuais.

A combinação dessas duas medidas permitiu a identificação de três tipos de critérios:

- Importante e consensual (IC): critério de avaliação consensualmente importante $\rightarrow$ incluir na imagem-objetivo;

- Não importante e consensual (NIC): critério consensualmente pouco importante $\rightarrow$ excluir da imagem-objetivo;

- Importante, mas não consensual (INC): critério importante, em que prevaleceu a discordância $\rightarrow$ excluir da imagem-objetivo.

Algumas considerações sobre a pontuação dos critérios feita pelos especialistas devem ser ressaltadas. O critério referente aos recursos humanos foi o mais valorizado, registrado nas pontuações atribuídas nas dimensões estrutural e assistencial. Os insumos contraceptivos foram o segundo critério de importância na dimensão estrutural, sendo a oferta ampliada de métodos contraceptivos o parâmetro de pontuação. Em relação à dimensão organizacional, a necessidade de sistematizar a assistência mediante a utilização do protocolo do planejamento familiar elaborado pelo gestor de saúde foi unânime, assim como a informatização do sistema de cadastro e atendimento do usuário que, por sua vez, pode subsidiar a avaliação do programa. A dimensão assistencial foi a que apresentou maior consenso entre os avaliadores.

Dentre os 57 critérios propostos na planilha da imagem-objetivo, quatro (7\%) não foram consensuais e três deveriam ser excluídos (5\%). Foi sugerida a inclusão de 13 critérios, sendo dez relativos aos aspectos estruturais e três referentes à dimensão organizacional.

Mediante tais resultados, verificou-se a necessidade de realizar reunião presencial com os avaliadores. Por intermédio da técnica de comitê tradicional 21, a imagem-objetivo do planejamento familiar foi rediscutida e, ao final, contemplou na dimensão estrutural 48 critérios; na organizacional quatro; e na assistencial oito, o que totalizou sessenta critérios. Cabe ressaltar que a dimensão estrutural foi a que obteve maior 
Estrutura

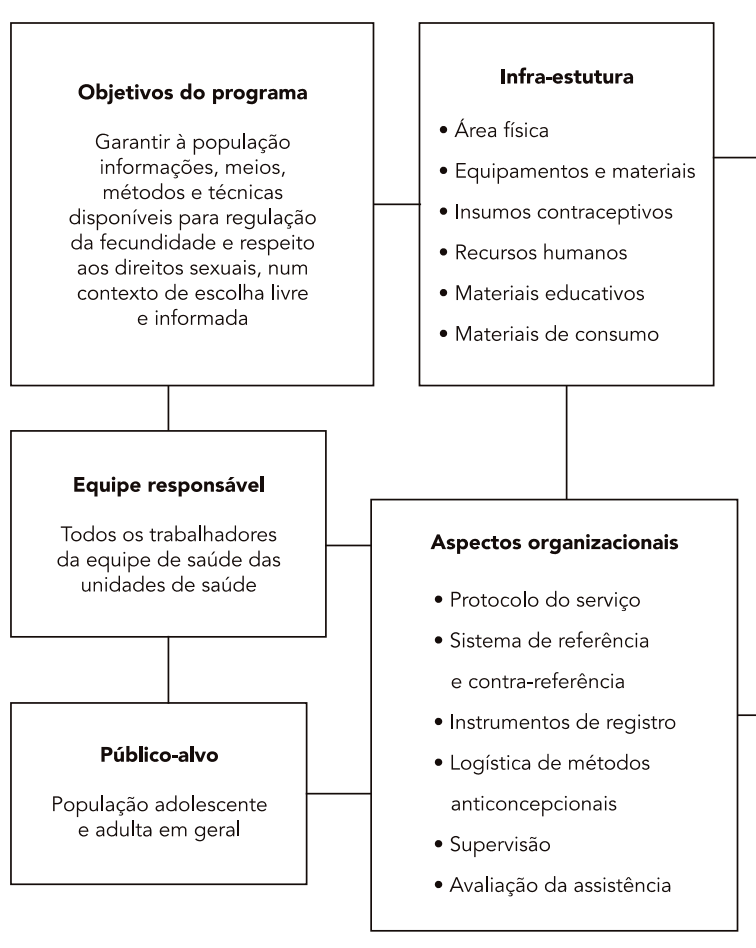

Processo

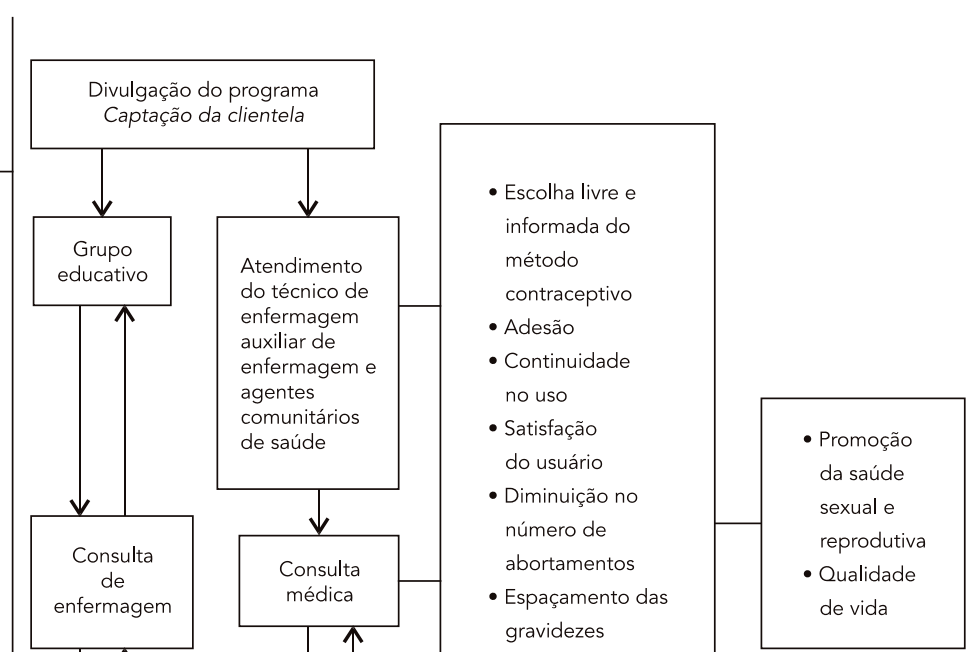

número de critérios por abranger cinco subdimensões com detalhamento dos elementos que a compuseram, acrescido dos dez critérios sugeridos pelos avaliadores.

A questão que suscitou polêmica referiu-se ao critério de peso para cada dimensão. Contudo, houve consenso de que a dimensão assistencial deveria ser a mais valorizada por representar o funcionamento efetivo do programa. A conferência também oportunizou discutir a pontuação e o grau de implantação para cada dimensão e subdimensão proposta na matriz de avaliação.

A Tabela 1 sumariza as dimensões, subdimensões, critérios e pontos estabelecidos na avaliação do grau de implantação do programa de planejamento familiar.

\section{Questionário de Avaliação de Serviços de Saúde Reprodutiva (QASAR)}

Baseado na literatura sobre atenção em planejamento familiar 1, protocolo de planejamento familiar do município 19 e importância relativa de cada dimensão e critério pautado nas discussões da conferência de consenso, elaborouse uma matriz de dimensões, subdimensões e critérios para avaliar o grau de implantação do programa de planejamento familiar nas 23 unidades básicas de saúde do município. Foram atribuídos cem pontos para a dimensão estrutural, cem pontos para a dimensão organizacional e duzentos pontos para a dimensão assistencial, totalizando quatrocentos pontos. Esses pontos foram distribuídos, pela pesquisadora, em cada subdimensão e critério e foram sumarizadas numa planilha, que constituiu o instrumento de avaliação do grau de implantação do programa de planejamento familiar.

O grau de aproximação entre a situação observada e a imagem-objetivo foi denominado grau de implantação, que correspondeu à diferença percentual entre a pontuação máxima e atribuída estimada para cada dimensão, subdimensão e critério de avaliação. A classificação do 
Dimensões, subdimensões, critérios e pontos estabelecidos na avaliação do grau de implantação do programa de planejamento familiar em 23 unidades básicas de saúde no Município de Maringá, Paraná, Brasil, 2006.

Pontos

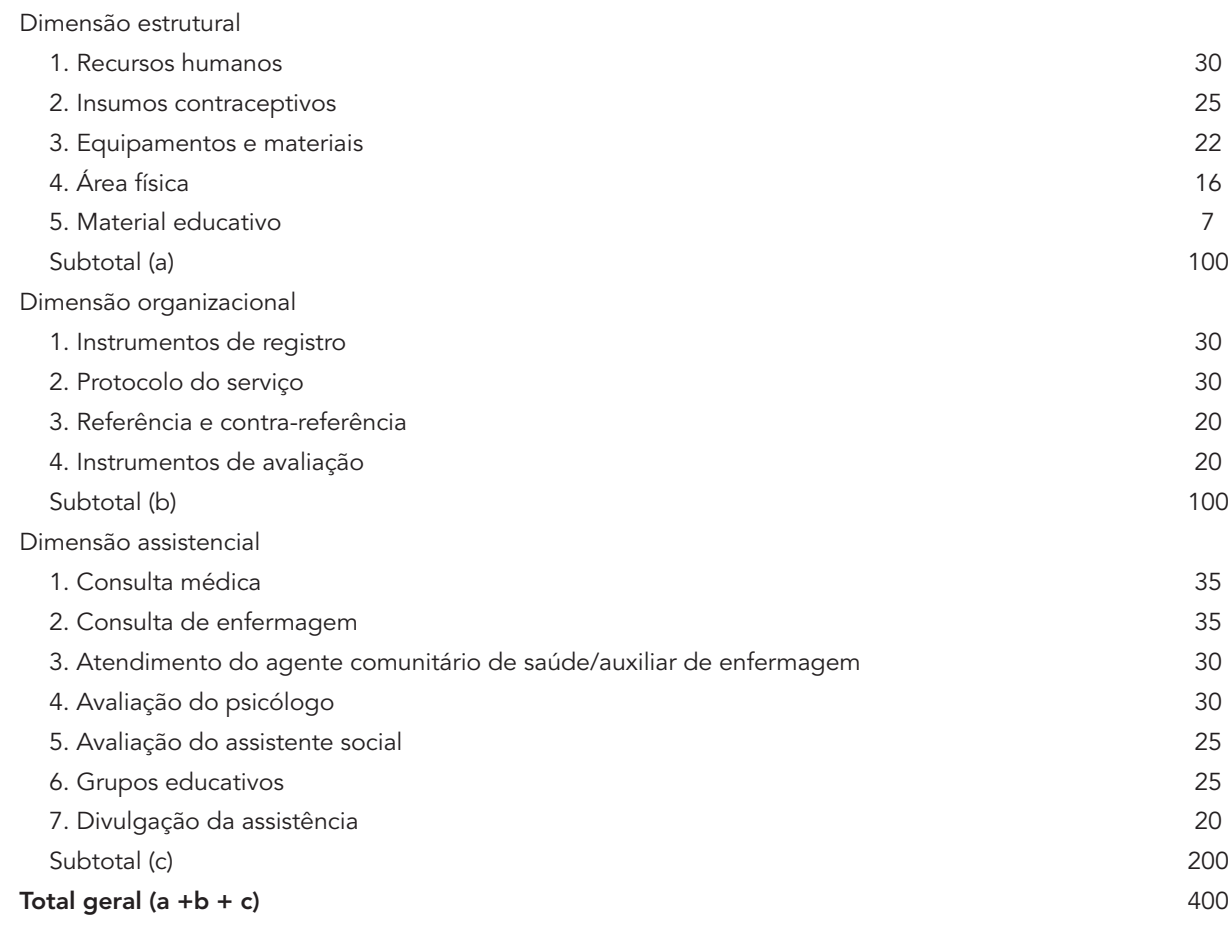

grau de implantação do programa de planejamento familiar foi calculada pela metodologia proposta por Silva et al. 22, que estabeleceu os seguintes pontos de corte, distribuídos em tercis: (1) incipiente: $\geq 0$ e $\leq 33,3 \%$; (2) intermediário: $>33,3$ e $\leq 66,6 \%$; (3) avançado: > 66,6\%.

Tomando-se por base os critérios de avaliação propostos na planilha de avaliação, foi elaborado um questionário auto-aplicável, que serviu de instrumento para coleta de dados nas 23 unidades básicas de saúde no Município de Maringá. Sendo assim, o Questionário de Avaliação de Serviços de Saúde Reprodutiva (QASAR), compreendeu o instrumento para coleta de dados com os respectivos critérios e pontuações que determinam o grau de implantação do programa de planejamento familiar para cada uma das dimensões e subdimensões analisadas.

\section{Aplicação do QASAR nas unidades básicas de saúde}

Três enfermeiras realizaram o trabalho de campo sob supervisão da pesquisadora. As 23 diretoras das unidades básicas de saúde responderam ao questionário após leitura e assinatura do termo de consentimento. A pesquisadora revisou os questionários e digitou os dados no programa SAS (SAS Inst., Cary, Estados Unidos), que foram analisados com auxílio do programa Statistica 6.0 (Statsoft Inc.; http://www.statsoft.com).

A pesquisa foi aprovada pelo Comitê de Ética em Pesquisa da Universidade Estadual de Maringá.

Para efeito da presente pesquisa, os resultados e discussão terão enfoque na análise do grau de implantação do programa segundo as três dimensões analisadas, bem como na inter-relação entre as mesmas.

\section{Resultados e discussão}

O grau de implantação do programa de planejamento familiar nas 23 unidades básicas de saúde, calculado pela somatória de pontos obtidos nas três dimensões - estrutural, organizacional e assistencial -, está representado na Figura 2. 
Distribuição do escore geral de pontos segundo grau de implantação do programa de planejamento familiar em 23 unidades básicas de saúde no Município de Maringá, Paraná, Brasil, 2006.

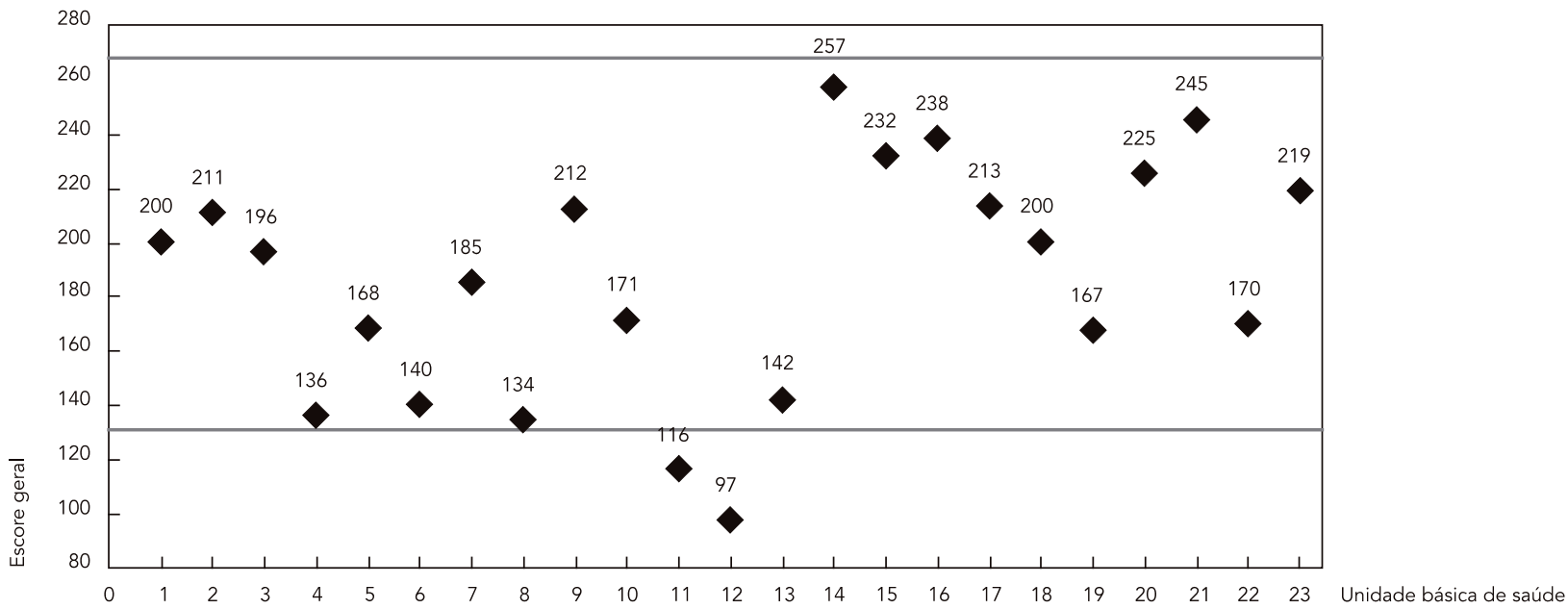

A média de escore foi de 186 pontos, com variação de 97 (unidade básica de saúde de número 12) a 257 pontos (unidade básica de saúde de número 14). A grande maioria das unidades básicas de saúde $(91,3 \%)$ obteve a classificação "intermediária” e duas $(8,7 \%)$ foram categorizadas como "incipientes" na implantação do programa de planejamento familiar. Nenhuma das unidades básicas de saúde obteve escore para ser considerada "avançada".

A diversidade de pontos registrados na classificação "intermediária" das 21 unidades básicas de saúde (de 134 a 257 pontos) chama atenção para os diferentes graus de implantação em cada uma das subdimensões e dimensões analisadas, que estão sumarizadas na Tabela 2.

Registrou-se variação de $66 \%$ a $87 \%$ no grau de implantação do planejamento familiar na dimensão estrutural, e a grande maioria das unidades básicas de saúde $(95,6 \%)$ obteve classificação "avançada" e apenas uma $(4,4 \%)$ foi considerada "intermediária" (unidade básica de saúde de número 11). As subdimensões área física e equipamentos foram as que obtiveram classificação "avançada” no grau de implantação (100\%) dentre todas as subdimensões e dimensões pesquisadas, fato que apontou condições adequadas para a implantação do programa quanto a esses dois quesitos.

Observou-se situação heterogênea de oferta dos insumos contraceptivos nas unidades bá- sicas de saúde, pois $52,2 \%$ das unidades foram consideradas "intermediárias" e 47,8\% "avançadas" na oferta dos contraceptivos. O anticoncepcional hormonal oral monofásico de baixa dosagem e o preservativo masculino foram os únicos insumos disponíveis em todas as unidades básicas de saúde. Por outro lado, o anticoncepcional oral de emergência estava disponível em 65,2\% das unidades básicas, o anticoncepcional hormonal injetável trimestral em $87 \%$, a minipílula em $95,6 \%$ e o preservativo feminino em $30,4 \%$ das unidades. A oferta do dispositivo intra-uterino variou em cada unidade básica de saúde e o anticoncepcional hormonal oral de média dosagem não constituía medicação padronizada pelo nível central e, por isso, não foi encontrado em nenhuma das unidades básicas de saúde.

Quanto aos recursos humanos, $74 \%$ das unidades básicas de saúde foram categorizadas como "avançadas" e 26\% "intermediárias" na implantação da equipe de saúde recomendada pelas normas do Ministério da Saúde 1. Mais da metade das unidades básicas de saúde (74\%) contava com 1 a 4 médicos capacitados e $26 \%$ delas, entre 5 a 7 . Todas as unidades dispunham de profissional enfermeiro capacitado, cujo número variou de 2 a 7 , com uma média de 3,5 enfermeiros por unidades básicas de saúde. Encontrou-se situação um pouco distinta em relação ao auxiliar de enfermagem, agente comunitário de saúde (ACS), psicólogo e assistente social. Pouco 
Grau de implantação (escore de pontos) segundo dimensão estrutural, organizacional e assistencial do programa de planejamento familiar em 23 unidades básicas de saúde no Município de Maringá, Paraná, Brasil, 2006.

\begin{tabular}{|c|c|c|c|c|c|c|c|c|c|c|c|}
\hline \multirow{2}{*}{$\begin{array}{l}\text { Dimensão } \\
\text { Sub-dimensão }\end{array}$} & \multicolumn{11}{|c|}{ Unidade básica de saúde } \\
\hline & 1 & 2 & 3 & 4 & 5 & 6 & 7 & 8 & 9 & 10 & 11 \\
\hline \multicolumn{12}{|l|}{ Estrutural } \\
\hline Área física & 16 * & 16 * & 16 * & 16 * & 13 * & 16 * & 16 * & 16 * & 16 * & 16 * & 16 * \\
\hline Equipamentos & $21 *$ & 19 * & 20 * & 22 * & 19 * & $21 *$ & 20 * & 19 * & 22 * & $21 *$ & 20 * \\
\hline Insumos & 20 * & 20 * & 17 * & $15^{\star \star}$ & $12 * \star$ & 12 *夫 & $13^{\star \star}$ & $13 * \star$ & 17 * & 18 * & $9 * *$ \\
\hline Recursos humanos & 18 ** & 24 * & 21 * & 18 ** & 18 ** & 24 * & 21 * & 18 ** & 30 * & 21 * & 21 * \\
\hline Material educativo & $0 * \star \star$ & 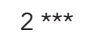 & $2 \star \star \star$ & $0 \star \star \star$ & 6 * & $2 \star \star \star$ & 5 * & $3 \star \star$ & $2 \star \star \star$ & $5 *$ & $0 * \star \star$ \\
\hline Subtotal (a) & 75 * & 81 * & 76 * & 71 * & 68 * & 75 * & 75 * & 69 * & 87 * & 81 * & 66 ** \\
\hline \multicolumn{12}{|l|}{ Organizacional } \\
\hline Contracepção cirúrgica & $0 * \star \star$ & $0 * \star \star$ & $0 * \star \star$ & $0 * \star \star$ & $0 * \star \star$ & $0 * \star \star$ & $10 * \star$ & $0 * \star \star$ & $0 * \star \star$ & $0 * \star \star$ & $0 * \star \star$ \\
\hline Instrumentos de registro & $10 * \star *$ & $10 \star \star \star$ & $10 \star \star \star$ & $10 * \star *$ & $10 \star \star \star$ & $10 \star \star \star$ & $10 * \star \star$ & $10 * \star \star$ & $10 \star \star \star$ & $10 \star \star \star$ & $10 * \star *$ \\
\hline Instrumentos de avaliação & $0 * \star \star$ & 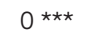 & $0 \star \star \star$ & $0 \star \star \star$ & $0 \star \star \star$ & $0 * \star \star$ & $0 * \star \star$ & $0 * \star \star$ & $0 * \star \star$ & $0 * \star \star$ & $0 * \star \star$ \\
\hline Protocolo serviço & $30 *$ & $15^{\star \star}$ & $15^{\star \star}$ & $15^{\star \star}$ & 15 ** & $0 * \star \star$ & $15 * \star$ & $0 * \star \star$ & $15^{\star \star}$ & $15^{\star \star}$ & $0 * \star \star$ \\
\hline Subtotal (b) & $40 * \star$ & $25 \star \star \star$ & $25^{\star \star \star}$ & $25 * \star *$ & $25 \star \star \star$ & $10 * \star \star$ & $35^{\star \star}$ & $10 \star \star \star$ & $25 \star \star \star$ & $25^{\star \star \star *}$ & $10 * \star *$ \\
\hline \multicolumn{12}{|l|}{ Assistencial } \\
\hline Divulgação & 20 * & $10 * \star$ & $0 * \star \star$ & $0 * \star \star$ & 20 * & $0 * \star \star$ & 20 * & $0 * * *$ & 20 * & 10 ** & $0 * \star \star$ \\
\hline Grupos educativos & $10 * \star$ & $10 * \star$ & $0 \star \star \star$ & $0 \star \star \star$ & $0 \star \star \star$ & $0 * \star \star$ & $0 \star \star \star$ & $0 \star \star \star$ & $0 * \star \star$ & $0 * \star \star$ & $0 \star \star \star$ \\
\hline Auxiliar de enfermagem/ & $15 * \star$ & 30 * & 30 * & $0 \star \star \star$ & $15^{\star \star}$ & $15 * \star$ & $15^{\star \star}$ & $0 * \star \star$ & $15^{\star \star}$ & $15 * \star$ & $0 \star \star \star$ \\
\hline \multicolumn{12}{|l|}{ Agente comunitário } \\
\hline \multicolumn{12}{|l|}{ de saúde } \\
\hline Consulta médica & 20 ** & 20 ** & 20 ** & 20 ** & 20 ** & 20 ** & 20 ** & 20 ** & 20 ** & 20 ** & 20 ** \\
\hline Consulta de enfermagem & 20 ** & 20 ** & 20 ** & 20 ** & 20 ** & 20 ** & 20 ** & 20 ** & 20 ** & 20 ** & 20 ** \\
\hline Psicóloga & $0 * \star \star$ & $15 * \star$ & $15^{\star \star}$ & $0 * \star \star$ & $0 * \star \star$ & $0 * \star \star$ & $0 * \star \star$ & $15^{\star \star}$ & $15 * \star$ & $0 * \star \star$ & $0 * \star \star$ \\
\hline Assistente social & $0 * \star \star$ & $0 \star \star \star$ & $10 * \star$ & $0 * \star \star$ & $0 \star \star \star$ & $0 * \star \star$ & $0 * \star \star$ & $0 * \star \star$ & $10 * \star$ & $0 * \star \star$ & $0 \star \star \star$ \\
\hline Subtotal (c) & $85^{* *}$ & $105 * \star$ & $95^{\star \star}$ & $40 * \star \star$ & $75^{\star \star}$ & $55^{\star \star \star}$ & $75^{\star *}$ & $55^{\star \star \star}$ & $100 * \star$ & 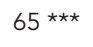 & 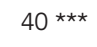 \\
\hline Total geral $(a+b+c)$ & $200 * \star$ & $211 \star \star$ & 196 ** & 136 ** & 168 ** & 140 ** & $185^{\star \star}$ & 134 ** & 212 ** & 171 ** & 116 *** \\
\hline
\end{tabular}

(continua)

mais da metade dos auxiliares de enfermagem e ACS $(52,1 \%)$ estava capacitado para realizar atendimento em planejamento familiar. Condição semelhante foi levantada em relação ao psicólogo. Apesar de a grande maioria das unidades disporem desse profissional (95,6\%), pouco mais da metade $(52,2 \%)$ recebeu capacitação específica na área. Seis unidades básicas de saúde (26\%) contavam com a assistente social na unidade e, entre elas, duas realizaram capacitação na área de saúde sexual e reprodutiva.

Em relação à disponibilidade de material educativo, encontraram-se diferentes graus de implantação, sendo 39,2\% das unidades básicas de saúde categorizadas como "incipiente", 30,4\%, "intermediária” e 30,4\% como "avançada”. Os recursos audiovisuais mais freqüentemente disponíveis foram o álbum seriado (34\%), o mostruário de métodos anticoncepcionais para visualização e manuseio do usuário (25\%) e figuras do aparelho reprodutor masculino e feminino $(19,1 \%)$.
Quanto à dimensão organizacional, concluiuse por um grau de implantação insatisfatório, pois, na classificação geral, $15(65,2 \%)$ unidades básicas de saúde obtiveram classificação "incipiente" e 8 (34,8\%) “intermediária”, com variação de $10 \%$ a $50 \%$ no grau de implantação.

O grau de implantação relativo ao critério referência para contracepção cirúrgica foi considerado "incipiente" em $74 \%$ das unidades básicas de saúde em razão da inexistência de mecanismo formal de encaminhamento para contracepção cirúrgica definitiva, e "intermediária" para $26 \%$ delas, pois o encaminhamento ocorreu por meio de ações individualizadas dos profissionais de saúde.

Apesar de 65\% das diretoras responderem que conheciam o protocolo de planejamento familiar elaborado pela coordenação do município ${ }^{19}$, poucos o utilizavam (17,4\%), e $47,8 \%$ o aplicavam "parcialmente", o que caracteriza implantação "intermediária” do protocolo para $65,2 \%$ 


\begin{tabular}{|c|c|c|c|c|c|c|c|c|c|c|c|c|}
\hline \multirow{2}{*}{$\begin{array}{l}\text { Dimensão } \\
\text { Sub-dimensão }\end{array}$} & \multicolumn{12}{|c|}{ Unidade básica de saúde } \\
\hline & 12 & 13 & 14 & 15 & 16 & 17 & 18 & 19 & 20 & 21 & 22 & 23 \\
\hline \multicolumn{13}{|l|}{ Estrutural } \\
\hline Área física & 13 * & 13 * & 16 * & 16 * & 16 * & 16 * & 16 * & 16 * & 16 * & $15 *$ & 12 * & 16 * \\
\hline Equipamentos & 19 * & 19 * & 20 * & $21 *$ & 18 * & 22 * & 22 * & 20 * & 22 * & 19 * & 22 * & 19 * \\
\hline Insumos & 14 ** & 17 * & 16 ** & 17 * & 16 ** & 17 * & $10 * \star$ & 14 ** & 19 * & $16 * *$ & 19 * & 20 * \\
\hline Recursos humanos & 21 * & 24 * & $18^{* *}$ & 24 * & 24 * & 24 * & 24 * & 21 * & 24 * & 30 * & $15^{* *}$ & 24 * \\
\hline Material educativo & $0 * \star \star$ & 4 ** & 7 * & 4 ** & $4^{\star \star}$ & 4 ** & $3 * \star$ & $1 * \star \star$ & 4 ** & 5 * & 7 * & 5 * \\
\hline Subtotal (a) & $67 *$ & 77 * & 77 * & 82 * & 78 * & 83 * & 75 * & 72 * & 85 * & 85 * & 75 * & 84 * \\
\hline \multicolumn{13}{|l|}{ Organizacional } \\
\hline Contracepção cirúrgica & $0 * \star \star$ & $0 \star \star \star$ & $10 * *$ & $0 * \star \star$ & $10 * *$ & 10 ** & $0 \star \star \star$ & $0 * \star \star$ & $0 * \star \star$ & 10 ** & $0 * \star \star$ & 10 ** \\
\hline Instrumentos de registro & $10 * \star \star$ & $10 \star \star \star$ & 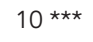 & 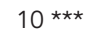 & $10 * * *$ & 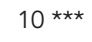 & 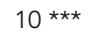 & 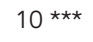 & $10 * \star \star$ & $10 * \star \star$ & $10 * \star \star$ & 10 *** \\
\hline Instrumentos de avaliação & $0 * \star \star$ & $0 \star \star \star$ & $0 \star \star \star$ & $0 \star \star \star$ & $0 * \star \star$ & $0 \star \star \star$ & $0 \star \star \star$ & $0 \star \star \star$ & $0 \star \star \star$ & $0 * \star \star$ & $0 \star \star \star$ & $0 * \star \star$ \\
\hline Protocolo serviço & $0 * \star \star$ & $15^{\star *}$ & 30 * & $15^{\star \star \star}$ & 30 * & $15^{* \star}$ & 30 * & $15^{\star \star *}$ & 15 ** & $15^{* \star}$ & $15^{\star \star}$ & $15^{\star \star}$ \\
\hline Subtotal (b) & $10 * * *$ & $25 \star \star \star$ & $50 * \star$ & $25^{\star \star \star *}$ & $50 * \star$ & $35 * \star$ & $40 * \star$ & $25 \star \star \star$ & $25^{\star \star \star *}$ & 35 ** & $25 \star \star \star$ & $35 * \star$ \\
\hline \multicolumn{13}{|l|}{ Assistencial } \\
\hline Divulgação & $0 * \star \star$ & $0 * \star \star$ & 20 * & 20 * & 20 * & $0 * \star \star$ & 20 * & 20 * & 20 * & 20 * & 20 * & 20 * \\
\hline Grupos educativos & $0 * \star \star$ & $0 \star \star \star$ & $10 * *$ & 25 * & $10 * \star$ & $10 * \star$ & $10 * *$ & $10 * \star$ & $10 * \star$ & $10 * \star$ & $10 * \star$ & $10 * *$ \\
\hline Auxiliar de enfermagem/ & $0 * \star \star$ & $0 \star \star \star$ & 30 * & $15 * \star$ & $15 * \star$ & 30 * & $15 * \star$ & $0 * \star \star$ & 30 * & 30 * & $0 \star \star \star$ & $15 \star \star$ \\
\hline \multicolumn{13}{|l|}{ Agente comunitário } \\
\hline Consulta médica & $0 * \star *$ & 20 ** & 35 * & 20 ** & 20 ** & 20 ** & 20 ** & 20 ** & 20 ** & 20 ** & 20 ** & 20 ** \\
\hline Consulta de enfermagem & $20 * \star$ & $20 * \star$ & $20 * \star$ & $20 * \star$ & $20 * \star$ & $20 * \star$ & $20 * \star$ & $20 * \star$ & $20 * \star$ & $20 * \star$ & $20 * \star$ & $20 \star \star$ \\
\hline Psicóloga & $0 * \star *$ & $0 * \star \star$ & $15^{\star *}$ & $15^{* *}$ & 15 ** & 15 ** & $0 * \star \star$ & $0 * \star \star$ & $15^{\star \star}$ & 15 ** & $0 * \star \star$ & $15^{\star \star *}$ \\
\hline Assistente social & $0 * \star *$ & $0 * \star \star$ & $0 \star \star \star$ & $10 * \star$ & 10 ** & $0 * \star \star$ & $0 * \star \star$ & $0 * \star \star$ & $0 \star \star \star$ & $10 * \star$ & $0 * \star \star$ & $0 * \star \star$ \\
\hline Subtotal (c) & $20 * \star \star$ & $40 \star \star \star$ & 130 ** & 125 ** & 110 ** & 95 ** & $85^{\star \star}$ & 70 ** & $115^{\star *}$ & 125 ** & 70 ** & 100 ** \\
\hline Total geral $(a+b+c)$ & $97 * \star \star$ & 142 ** & $257 \star \star$ & 232 ** & 238 ** & 213 ** & 200 ** & 167 ** & 225 ** & $245^{\star \star}$ & 170 ** & 219 ** \\
\hline
\end{tabular}

\footnotetext{
* Avançado;

** Intermediário;

*** Incipiente.
}

das unidades. Quatro diretoras $(17,4 \%)$ desconheciam a existência de protocolo (implantação "incipiente") e somente quatro $(17,4 \%)$ relataram seguir suas normativas (grau de implantação “avançada”).

Quanto aos instrumentos de registro, todas as unidades registravam as atividades de planejamento familiar somente no prontuário do paciente, o que caracterizou grau de implantação "incipiente". Categorização semelhante refere-se aos instrumentos de avaliação do programa, já que nenhuma das entrevistadas realizava avaliação do programa de planejamento familiar, situação que denota a ausência deste instrumento de gestão nas unidades básicas de saúde.

Quanto à dimensão assistencial, 16 (69,6\%) unidades básicas de saúde foram classificadas na categoria "intermediária" e sete $(30,4 \%)$ "incipiente”, com variação de $10 \%$ a $65 \%$ no grau de implantação das ações do planejamento familiar.

A análise conjunta das sete subdimensões contidas na dimensão assistencial (Tabela 2) ex- plicita a heterogeneidade de ações e a ausência de sistematização da atenção.

A estratégia de captação da clientela por intermédio da divulgação da assistência não ocorreu em 34,8\% das unidades básicas de saúde, o que caracterizou grau de implantação "incipiente". Em 8,7\% das unidades, a divulgação foi realizada somente quando o usuário buscou informações na unidades básicas de saúde (implantação "intermediária”) e, em 56,5\% dos casos, a divulgação efetivou-se por intermédio das equipes do PSF, grupo de gestantes, na comunidade (implantação “avançada”).

O Ministério da Saúde ${ }^{1}$ recomenda que as ações educativas em contracepção devam ser realizadas em grupo, procedendo à primeira consulta médica, a fim de que os usuários compartilhem dúvidas, sentimentos e conhecimentos.

$\mathrm{Na}$ análise do grau de implantação dos grupos educativos de planejamento familiar, registrou-se que $47,8 \%$ das unidades básicas de saúde foram categorizadas como "incipientes", uma vez que a atividade não era oferecida na unidade, e 
em 47,8\% "intermediária" (o tema do planejamento familiar foi abordado em outros grupos educativos: gestantes, adolescentes e mães). Apenas uma unidade promovia, no mínimo uma vez ao mês, grupos educativos sobre planejamento familiar, além da incorporação do tema por parte dos grupos (grau de implantação "avançada”).

Partindo-se da premissa de que a atividade educativa em grupo deve ser a porta de entrada para o atendimento sistematizado, identificaram-se deficiências que se originaram na captação do usuário, persistiram nos grupos educativos e se estenderam à atenção subseqüente da equipe de saúde.

Em relação ao atendimento individual realizado pela equipe de saúde, registrou-se que a subdimensão referente à consulta de enfermagem foi classificada como "intermediária" em todas as unidades básicas de saúde, vale dizer, a prática assistencial do enfermeiro, exercida na consulta de enfermagem, foi realizada conforme demanda espontânea, para orientação, distribuição de contraceptivos e encaminhamentos.

Categorização semelhante foi encontrada na consulta médica porque $21(91,4 \%)$ unidades básicas de saúde foram consideradas "intermediárias" nesta subdimensão, ou seja, a consulta médica de planejamento familiar era realizada conforme demanda espontânea para prescrição de contraceptivos e encaminhamentos.

Apesar de a quase totalidade das unidades básicas de saúde contar com o psicólogo (95,6\%), a atuação deste profissional foi considerada "incipiente" em 12 (52,1\%) unidades básicas de saúde, pois o profissional não realizava atendimento em planejamento familiar e, em 11 (47,9\%) unidades, o profissional realizava atendimento conforme demanda espontânea, o que caracterizou implantação "intermediária". Em relação ao atendimento da assistente social, encontrou-se que 18 (78,2\%) unidades básicas de saúde situavam-se na categoria "incipiente" e $5(21,7 \%)$ na condição de "intermediária”. Estas informações traduziram a baixa inserção dos profissionais que, de certa forma, era esperada, uma vez que $47,8 \%$ dos psicólogos das unidades básicas de saúde não foram capacitados na área de planejamento familiar, e apenas seis unidades básicas de saúde contavam com a assistente social.

Em contraste com esses dados, encontra-se a atuação do auxiliar de enfermagem e do ACS. A atuação destes profissionais foi expressiva, à medida que mais da metade $(69,5 \%)$ alcançou o grau de implantação "intermediária" (43,5\%) na atenção e $26 \%$ foi categorizada "avançada". Considerando que pouco mais da metade $(52,1 \%)$ foi capacitada para realizar atendimento em planejamento familiar, os dados revelam a forte inserção de tais profissionais na atenção, bem como reforçam a necessidade de investir em sua capacitação.

Em seu conjunto, a avaliação do grau de implantação na dimensão assistencial apontou para um modelo de atenção baseado no atendimento da demanda espontânea e, sobretudo, que restringe o direito do usuário de exercer amplamente seus direitos sexuais e reprodutivos, à proporção que limita o direito de escolha livre e informada do método contraceptivo e a oportunidade de discutir suas necessidades e opções em relação à fertilidade e à contracepção.

A Figura 3 sumariza o grau de implantação do programa de planejamento familiar nas 23 unidades básicas de saúde, com enfoque na interrelação entre as três dimensões.

Na Figura 3, chama a atenção o grau de implantação "avançado" na dimensão estrutural, em contraste com as dimensões organizacional e assistencial, nas quais a heterogeneidade no grau de implantação "incipiente" e "intermediário" caracteriza as 23 unidades de saúde. Verificouse também a relação entre o grau de implantação nas dimensões organizacional e assistencial, pois os resultados insatisfatórios verificados na dimensão organizacional refletiram-se na implantação da assistência e vice-versa.

Vuori 23 , ao discutir as abordagens para avaliação da qualidade do cuidado proposta por Donabedian ${ }^{24}$, ressalta que, na atenção à saúde, a estrutura está claramente afastada do resultado, dado que mesmo as melhores pré-condições podem ser mal usadas, enquanto a excelência profissional pode resultar em benefícios, mesmo em precárias condições de trabalho. $\mathrm{O}$ autor infere que apesar de uma boa estrutura não garantir resultado favorável, ela pode prevenir contra a deterioração da qualidade que poderia ocorrer, caso não houvesse padrões mínimos ou outras proteções estruturais.

Sobre esta questão, Scochi 25 complementa que contabilizar e analisar os recursos são relevantes no planejamento, mas, enquanto medida para avaliar qualidade, tem menos importância que os componentes de processo e de resultado. Segundo a autora, deficiências estruturais podem, de fato, implicar baixa qualidade, mas estrutura adequada não implica necessariamente alta qualidade: apenas aponta que possui potencial para isso.

Infere-se que essas afirmações aplicam-se a esta pesquisa avaliativa, visto que $95,6 \%$ das unidades básicas de saúde foram classificadas como "avançadas" no grau de implantação da dimensão estrutural e nenhuma delas alcançou este escore na avaliação da dimensão assistencial. Isso pode 
Distribuição do escore geral de pontos segundo grau de implantação e dimensão estrutural, organizacional e assistencial do programa de planejamento familiar em 23 unidades básicas de saúde no Município de Maringá, Paraná, Brasil, 2006.

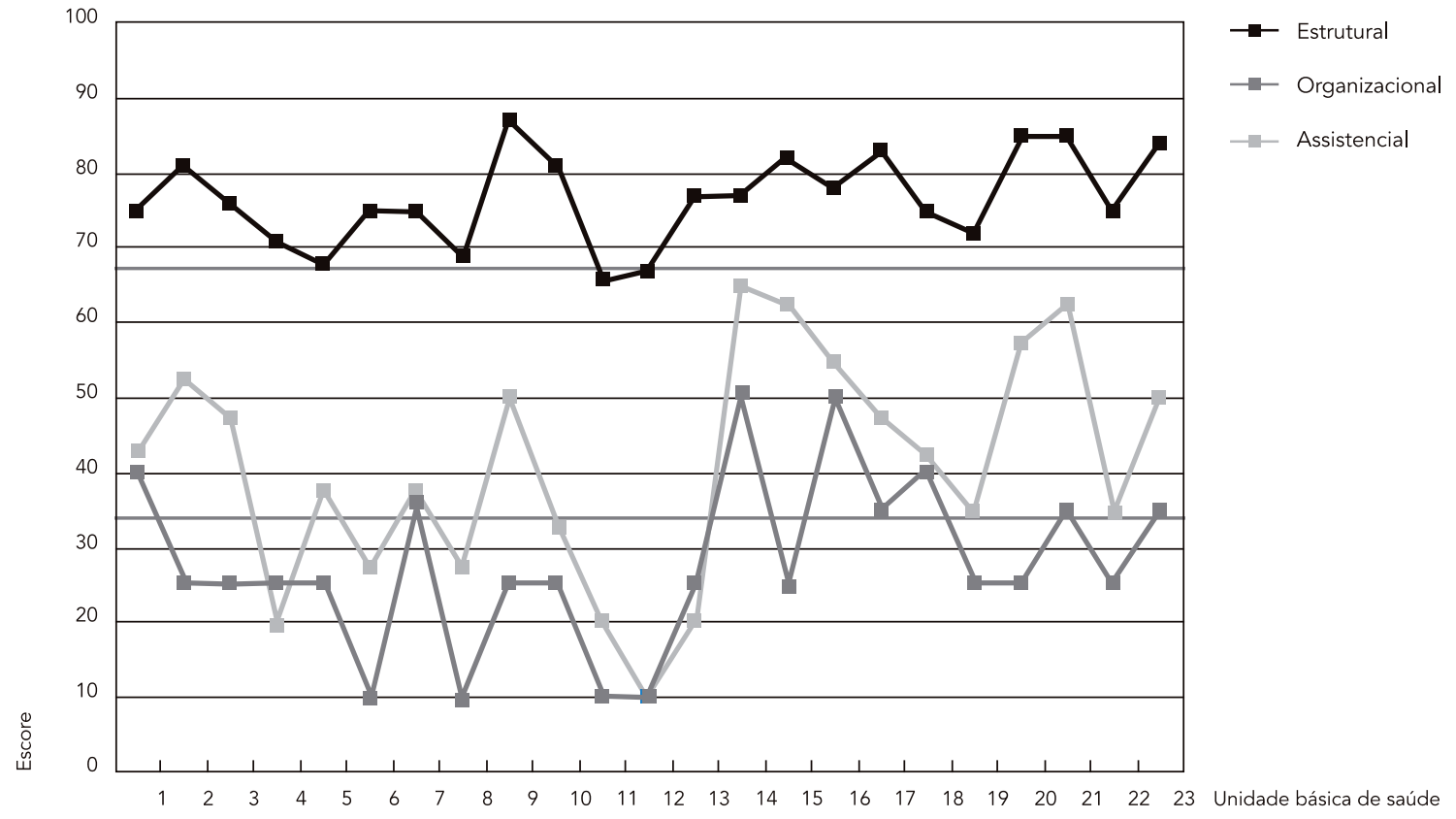

indicar que o grau de implantação do programa na dimensão estrutural tem pouca influência sobre o grau de implantação da dimensão assistencial, pois esta depende de uma multiplicidade de fatores ligados a recursos humanos, relações interpessoais, capacitação profissional, aspectos organizacionais, entre outros. Por outro lado, o resultado pode ser encarado com otimismo, já que as unidades básicas de saúde apresentaram condições estruturais potencialmente favoráveis à implantação da assistência.

\section{Considerações finais}

Segundo Teixeira \& Vilasboas 26 , a prática cotidiana de gestão do SUS, em qualquer nível de governo, coloca um enorme desafio aos gestores, que é o de identificar e selecionar os métodos, técnicas e instrumentos de trabalho que o ajudem a tomar decisões e a conduzir o processo de implementação das políticas, programas e ações de saúde sob sua responsabilidade.

Considerando a complexidade da produção de ações e serviços de saúde, o QASAR consti- tui ferramenta para avaliar programas de saúde reprodutiva, à medida que proporciona visão detalhada e integral do grau de implantação do programa de planejamento familiar e permite identificar as dificuldades e aspectos facilitadores para implantação do programa, reveladas nas dimensões e subdimensões. Constitui instrumento aplicável aos processos de planejamento e gestão dos serviços de saúde, como ferramenta destinada a intervir sobre os serviços de saúde reprodutiva, a fim de melhorar sua pertinência, efetividade e eficiência.

A pesquisa avaliativa revelou um modelo de atenção marcado pela heterogeneidade de ações e ausência de sistematização da assistência. A medicalização da atenção foi observada na oferta limitada e heterogênea de insumos contraceptivos, no atendimento baseado na demanda espontânea para distribuição de contraceptivos e, particularmente, na implantação incipiente de práticas educativas em saúde sexual e reprodutiva, que restringem a escolha livre e informada do método anticoncepcional.

A grande maioria das unidades básicas de saúde $(91,3 \%)$ foi classificada como "intermedi- 
ária” na implantação do programa de planejamento familiar, $8,7 \%$ foram categorizadas como "incipientes" e nenhuma obteve escore para ser considerada "avançada". Chamou a atenção o grau de implantação "avançado" na dimensão estrutural, em contraste com as dimensões organizacional e assistencial, nas quais a heterogeneidade no grau de implantação "incipiente" e "intermediário" caracterizou as unidades básicas de saúde.

Evidenciaram-se aspectos que dificultaram a implantação do programa, identificados na oferta limitada de materiais educativos; inexistência de mecanismo formal de encaminhamento para contracepção cirúrgica definitiva; desconhecimento ou pouca utilização do protocolo de planejamento familiar elaborado pelo município; ausência de ações de avaliação do programa; falta de divulgação da assistência; pouca oferta de grupos educativos sobre planejamento familiar; consulta médica e de enfermagem destinada à prescrição, distribuição de insumos contraceptivos e encaminhamentos; baixa inserção dos profissionais psicólogo e assistente social no atendimento em planejamento familiar e a capacitação desigual da equipe de saúde.

\section{Resumo}

O objetivo deste estudo foi desenvolver um instrumento para avaliar a implantação da assistência em contracepção em serviços de saúde, bem como aplicálo nas 23 unidades básicas de saúde no Município de Maringá, Paraná, Brasil. Elaborou-se o modelo teórico-lógico, correspondente a uma "imagem-objetivo" do programa de planejamento familiar. Por meio da técnica Delphi e conferência de consenso, seis especialistas validaram a imagem-objetivo do programa, que contemplou três dimensões e 60 critérios de avaliação. Elaborou-se um instrumento para coleta de dados e uma planilha para avaliar o grau de implantação do programa de planejamento familiar, que constituíram $o$ Questionário de Avaliação de Serviços de Saúde Reprodutiva (QASAR). A grande maioria das unidades básicas de saúde $(91,3 \%)$ recebeu a classificação "intermediária" na implantação do programa de planejamento familiar, 8,7\% foram categorizadas "incipientes" e nenhuma obteve escore para ser considerada "avançada". O grau de implantação "avançado" na dimensão estrutural contrastou com as dimensões organizacional e assistencial. O instrumento constitui ferramenta para avaliar programas de saúde reprodutiva, aplicável aos processos de planejamento e gestão dos serviços de saúde.

Gestão em Saúde; Serviços de Saúde Reprodutiva; Avaliação em Saúde; Planejamento Familiar
O estudo apontou que as dificuldades para implantação do programa concentraram-se na dimensão organizacional e assistencial, a saber, que envolvem o processo da atenção. Considerando que a maioria das unidades básicas de saúde apresenta condições estruturais favoráveis à implantação do programa, mas que, sozinhas, não garantem sua implantação, é necessário que gestores e equipe de saúde reflitam e discutam sobre o processo de trabalho da equipe e elaborem estratégias para a implantação efetiva do programa, tendo por princípio norteador o respeito aos direitos sexuais e reprodutivos e à integralidade da atenção.

Pontuam-se algumas questões que fugiram aos objetivos deste trabalho e que poderão ser discutidas no âmbito da gestão do serviço de saúde e exploradas em pesquisas futuras, quais sejam: a importância e o significado das ações de planejamento familiar na esfera da saúde sexual e reprodutiva e a atuação do profissional de saúde nas ações de planejamento familiar.

Finalmente, recomenda-se a reavaliação posterior do programa de planejamento familiar no município, mediante aplicação do QASAR, bem como sua aplicação em outros serviços de saúde.

\section{Agradecimentos}

Ao 12o Programa Interinstitucional de Treinamento em Metodologia de Pesquisa em Gênero, Sexualidade e Saúde Reprodutiva/Fundação Ford que proporcionou apoio técnico, logístico e financeiro para o desenvolvimento da pesquisa. Aos coordenadores, docentes, assistentes de coordenação e servidores técnico-administrativos do Instituto de Saúde Coletiva, Universidade Federal da Bahia (ISC/UFBA), Núcleo de Estudos de População, Universidade Estadual de Campinas (NEPO/ UNICAMP) e Instituto de Saúde, São Paulo. Às colegas participantes do Programa, pelo carinho e amizade. Aos especialistas participantes da conferência de consenso: Deise Serafim, Regina Lúcia Dalla Torre Silva, Maria Tereza Soares Rezende Lopes, Maria da Penha Sapata, Nelson Shozo Uchimura e Adilson Carlos Gomes pelas valiosas contribuições. A Carleno Alcides Amorim Quintino, pelo apoio estatístico. À Secretaria Municipal de Saúde de Maringá e diretoras de saúde, que se prontificaram a participar da pesquisa. Ao Hospital Universitário de Maringá, pelo apoio e incentivo. 


\section{Referências}

1. Ministério da Saúde. Planejamento familiar: manual para o gestor. Brasília: Ministério da Saúde; 2002.

2. Fundo de População das Nações Unidas. Situação da população mundial 2004. http://www.onu-bra sil.org.br/documentos_estudos.php (acessado em 08/Set/2006).

3. Secretaria Nacional de Programas Especiais de Saúde. Assistência integral à saúde da mulher: bases de ação programática. Brasília: Centro de Documentação, Ministério da Saúde; 1984

4. Ministério da Saúde. Saúde, Brasil. Brasília: Ministério da Saúde; 2005.

5. Díaz J, Díaz M. Qualidade de atenção em saúde sexual e reprodutiva: estratégias para mudanças. In: Galvão L, Díaz J, organizadores. Saúde sexual e reprodutiva no Brasil: dilemas e desafios. São Paulo: Editora Hucitec; 1999. p. 209-33.

6. Espejo X, Tsunechiro A, Osis MJ, Duarte G, Bahamondes L, Sousa MH. Adequação dos conhecimentos sobre métodos anticoncepcionais entre mulheres de Campinas, São Paulo. Rev Saúde Pública 2003; 37:583-90.

7. Vieira EM. Políticas públicas e contracepção no Brasil. In: Berquó E, organizadora. Sexo \& vida: panorama da saúde reprodutiva no Brasil. Campinas: Editora da Unicamp; 2003. p. 151-96.

8. Moreira MHC, Araújo JNG. Planejamento familiar: autonomia ou encargo feminino? Psicol Estud 2004; 9:389-98

9. Paniz VMV, Fassa AG, Silva MC. Conhecimento sobre anticoncepcionais em uma população de 15 anos ou mais de uma cidade do Sul do Brasil. Cad Saúde Pública 2005; 21:1747-60.

10. Souza JMM, Pelloso SM, Uchimura NS, Souza F. Utilização de métodos contraceptivos entre usuárias da rede pública de saúde do município de Maringá-PR. Rev Bras Ginecol Obstet 2006; 28:271-7.

11. Costa AM, Guilhem D, Silver LD. Planejamento familiar: a autonomia das mulheres sob questão. Rev Bras Saúde Matern Infant 2006; 6:75-84.

12. Schor N, Ferreira AF, Machado VL, França AP, Pirotta KCM, Alvarenga AT, et al. Mulher e anticoncepção: conhecimento e uso de métodos anticoncepcionais. Cad Saúde Pública 2000; 16:377-84.

13. Souza JMM. Avaliação da saúde reprodutiva das usuárias da rede pública de saúde do município de Maringá-PR [Dissertação de Mestrado]. Maringá: Universidade Estadual de Maringá; 2006.

14. Taguchi WS, Nóbrega MGG, Santos JH, Roncada EVM, Nakazora DY, Nagahama EEI, et al. Características dos homens submetidos à vasectomia no serviço de urologia do Departamento de Medicina da Universidade Estadual de Maringá, Maringá, Estado do Paraná. Acta Sci Health Sci 2005; 27 : 189-93.

15. Osis MJD, Faúndes A, Makuch MY, Mello MB, Sousa MH, Araújo MJO. Atenção ao planejamento familiar no Brasil hoje: reflexões sobre os resultados de uma pesquisa. Cad Saúde Pública 2006; 22:2481-90.
16. McKinlay JB. More appropriate evaluation methods for community - level health interventions (introduction to the special issue). Eval Rev 1996; 20:237-43.

17. Hartz ZMA, Champagne F, Contandriopoulos AP, Leal MC. Avaliação do programa materno-infantil: análise de implantação em sistemas locais de saúde no Nordeste do Brasil. In: Hartz ZMA, organizadora. Avaliação em saúde: dos modelos conceituais à prática na análise da implantação de programas. Rio de Janeiro: Editora Fiocruz; 1997. p. 89-131.

18. Secretaria Municipal de Saúde. Assessoria de planejamento. Plano municipal de saúde 2006-2007. Maringá: Secretaria Municipal de Saúde; 2006.

19. Barreto CP. Protocolo de planejamento familiar. Maringá: Coordenação da Saúde da Mulher, Secretaria Municipal de Saúde; 2004.

20. Medina MG, Silva GAP, Aquino R, Hartz ZMA. Uso de modelos teóricos na avaliação em saúde: Aspectos conceituais e operacionais. In: Hartz ZMA, Silva LMV, organizadoras. Avaliação em saúde: dos modelos teóricos à prática na avaliação de programas e sistemas de saúde. Salvador: EDUFBA/Rio de Janeiro: Editora Fiocruz; 2005. p. 41-63.

21. Souza LEPF, Silva LMV, Hartz ZMA. Conferência de consenso sobre a imagem-objetivo da descentralização da atenção à saúde no Brasil. In: Hartz ZMA, Silva LMV, organizadoras. Avaliação em saúde: dos modelos teóricos à prática na avaliação de programas e sistemas de saúde. Salvador: EDUFBA/Rio de Janeiro: Editora Fiocruz; 2005. p. 65-102.

22. Silva LMV, Hartz ZMA, Chaves SCM, Silva GAP. Metodologia para análise da implantação de processos relacionados à descentralização da atenção à saúde no Brasil. In: Hartz ZMA, Silva LMV, organizadoras. Avaliação em saúde: dos modelos teóricos à prática na avaliação de programas e sistemas de saúde. Salvador: EDUFBA/Rio de Janeiro: Editora Fiocruz; 2005. p. 207-53.

23. Vuori HI. A qualidade da saúde. Divulg Saúde Debate 1991; 30:17-25.

24. Donabedian A. Explorations in quality assessment and monitoring. The definitions of quality and approaches to its assessment. Ann Harbor: Health Administration Press I; 1980.

25. Scochi MJ. Municipalização e avaliação de qualidade de serviços de saúde: uma análise localizada [Tese de Doutorado]. Rio de Janeiro: Escola Nacional de Saúde Pública, Fundação Oswaldo Cruz; 1996.

26. Teixeira C, Vilasboas AL. Planejamento e gestão de sistemas e serviços de saúde. http://www.itd. bvs.br/itd-mod/public/scripts/php/page_show_ index.php (acessado em 27/Mar/2007).

Recebido em 15/Jan/2008

Versão final reapresentada em 24/Abr/2008

Aprovado em 19/Mai/2008 